\title{
Unmet need: specific pcos-diagnostic
}

\begin{abstract}
Background: One of the most common endocrine and metabolic disorders found in women in reproductive age, affecting-depending kind of publication-5 to $12 \%$ of women worldwide is the Polycystic Ovarian Syndrome (PCOS). It is suggested that only $50 \%$ of them are diagnosed and the grey zone of affected women might be much higher. Because of the missing specific diagnostics except the Rotterdam Criteria and focus on treatment of symptoms patients must suffer much too long until they get diagnosed exactly.
\end{abstract}

Method: This is a Short Review about unmet diagnostically needs to detect PCOS and experiences out of case studies and a PubMed enquiry using the search criteria "PCOS diagnostic method, 2016-2017, human, clinical trials".

Result: 33 hits were found but no specific diagnostic method was described. Since 1990 the Rotterdam Criteria has been defined the leading characteristics to diagnose PCOS, there is no improvement known. Followed by visual factors as hirsutism, acne, thin hairline, body fat distribution there is no specific diagnostic for PCOS in lean or overweight/obese patients until wish to conceive available.

Conclusion: It must be the aim in future to detect PCOS at an early stage to enable an acceptable life for these women by clarity and reduction of several health risk factors as e.g. type-2-diabetes, cardio vascular disease, and hypercholesterolemia. Diagnostically needs must be determined, measures be defined which should support/ replace the Rotterdam Criteria. And there is another phenotype, the lean women with morphological existent polycystic ovaries but neither clinical symptoms nor biochemical spillover of androgen.

Keywords: PCOS, rotterdam criteria, diagnostic, lean, obese/overweight
Volume 2 Issue I - 2018

Marion Eckert Krause

Fachinstitut für Stoffwechsel und Gesundheit,Austria

Correspondence: Marion Eckert Krause, Fachinstitut für Stoffwechsel und Gesundheit, A-6800 Feldkirch,Am Oberen Riegel 42 b, Austria, Tel +43-650-85I-7993,

Emailmek@eckertkrause.com

Received: January 12, 2018 | Published: February 07, 2018
Abbreviations: PCOS, polycystic ovary syndrome; BMI, body mass index; $\mathrm{RC}$, rotterdam criteria

\section{Introduction}

The PCOS diagnosis based on exclusion of causes, such as androgenproducing neoplasm, hyperprolactinemia, adult-onset congenital adrenal hyperplasia. ${ }^{1}$ It is characterized by oligoor anovulation and Clinical and/or biochemical signs of hyperandrogenism Polycystic ovaries (by ultrasound), hirsutism, and infertility and followed since 1990 the Rotterdam Classification: ${ }^{2}$

i. PCOS impacts: skin, hair, body hair, body shape, body smell, power, sexuality/sexiness, partnership, emotion, selfconfidence, metabolism, fertility, social life

ii. PCOS could lead to: depression thought of suicide, desperation, loneliness, exclusion, frustration, aggression/auto-aggression, binge eating, bulimia, anorexia, drugs and alcohol abuse.

While there are no obvious signs if a woman is suffering of «PCOS», the patient herself already feels that something is going «wrong». From puberty to menopause this syndrome without clear root cause is present, in lean women with BMI $<25 \mathrm{Kg} / \mathrm{m}^{2}$ as well as in overweight/obese women. PCOS became diagnosed approx. at the age $20 .^{3}$ At that point a lot of unsureness over a couple of years was with the women, also related to wasted years for lifestyle adaption. ${ }^{4}$

Some other facts why it is not that easy to diagnose PCOS: Women do not report their symptoms; they do hair removal and deny the symptoms. Women reflect to their family and find the explanation "normal". Doctors are not well trained about PCOS and have the association of overweight/obese more than on lean patients. Doctors treat symptoms separately. ${ }^{5}$ There is no or not sufficiently collaboration among gynecologists, endocrinologists, dermatologists, pediatrics. Diagnostic do not meet the needs for PCOS, there is no quick test (e.g. as for pregnancy) available. PCO-Syndrome is based on exclusion criteria (Rotterdam Criteria). Characteristics can vary, e.g. body weight, eating habits, common lifestyle as well as oral contraceptive which have impact on test results (e.g. lower androgen, decrease body hair).

\section{Review case study with questionnaire}

A young woman answered, that her gynecologist spoke about PCOS after the examination of the ovaries and a high LH-value. Patient-age 23, body mass index (BMI) $20 \mathrm{~kg} / \mathrm{m}^{2}$, doing sport, balanced eating, moderate alcohol consumption and smoking. In 2017 a laparoscopy resulted in sclerotic, polycystic ovaries. No hirsutism but thin hair and regions of alopecia could be stated. Noticed first changes in 2016 when the period became irregular and very painful with the impression of swollen ovaries. No other diseases are known. Family anamnesis showed type 2 diabetes, coronary heart disease and depression within the males. Patient did not use drugs or other medical treatment. She lives in a psychological trauma situation related to insufficient diagnostic possibilities to show the severity of her PCOS. This has impact on social life, partnership and family. To learn about conceiving in future was postponed by her doctor because of her young age. She felt ignored and frustrated, changed the doctor but the experience was nearly the same. She got additional 
information by coach, internet platforms and self-help group, less or not understandable by her doctor.

\section{Review case study with interview}

Patient-age 28, academic scientist, high acceptance in public/job, BMI 24, lean but typical body shape of PCOS with smaller upper part of the body than girth of hips, athletic muscle structure, no hirsutism, clean skin, thin hairline. No diseases in family anamnesis, no overweight with parents. Problems she mentioned were with eating and calorie restriction because of the rapid weight gain experience and on common psychological feelings. She described her PCOS journey as followed: First menstruation with age 12. Birth control pill administered at age 18. Start of problems was at age 20 based also on experiments with diverse pill types over 11 months; no menstrual bleeding, no cycle activity but fast weight gaining. Then she became ultrasound-diagnosed with PCOS, no explanation, no tests, no further investigations were done. Her weight focused and restricted lifestyle lead to psychological problems, e.g. eating disorder, daily athletic sports, afraid to start a partnership. Her information she got by internet platforms and less by her doctors.

\section{Review case study with DEBEC-method}

Patient, age 25, journey of different birth control pills, stop taking the pill and learning about rapid weight gain, hirsutism, skin and hair disorder, diagnosed at the age of 20 by Rotterdam Criteria, blood test and ultrasound on demand, psychological burden determined in depression, desperation, loneliness and a certain feeling not to be a normal women and able to conceive. Work with a health coach and reduction of $20 \mathrm{~kg}$ weight. Her information she got by her coach and via internet platforms, less or not understandable by her doctors. She missed tests to follow up the state of PCOS.

\section{Limitations}

This short review in combination of three summed up case studies could not assure a complete view on the diagnostic landscape. There is no diagnostic guideline as reference available.

\section{Conclusion}

The different case studies show a typical picture of the situation of women with PCOS and meet the result of the review that there is no specific and structured diagnostic guideline available. An early specific diagnose will support lifestyle modification, health risk mitigation and lowering psychological factors. Related to the decades of processing with Rotterdam Criteria based on still unknown root cause modern diagnostic should meet the needs e.g. with specific tests/quick tests. Development of diagnostic methods and further structured studies are needed.

\section{Acknowledgements}

I would like to thank my group of clients who enabled me to understand the unmet needs within diagnostically requirements and the actual situation.

\section{Conflicts of interest}

The author declares no conflict of interest.

\section{References}

1. Cahill D. PCOS. Clin Evid. 2009;15:1408.

2. Roe AH, Dokras A. The Diagnosis of Polycystic Ovary Syndrome in Adolescents. Rev Obstet Gynecol. 2011;4(2):45-51.

3. Carmina E, Campagna AM, Lobo RA. A 20-year follow-up of young women with polycystic ovary syndrome. Obstet Gynecol. 2012;119(2 Pt 1):263-269.

4. Legro RS, Arslanian SA, Ehrmann DA, et al. Diagnosis and Treatment of Polycystic Ovary Syndrome: An Endocrine Society Clinical Practice Guideline. The Journal of Clinical Endocrinology \& Metabolism. 2013;98(12):4565-4592.

5. Goyal M, Dawood AS. Debates regarding lean patients with polycystic ovary syndrome: a narrative Review. J Hum Reprod Sci. 2017;10(3):154 161. 